\title{
基于红外图像的绝缘子提取方法
}

\author{
赵利鸿，高 强，于 晓，李大华 \\ (天津理工大学 电气电子工程学院, 天津市复杂系统控制理论及应用重点实验室, 天津 300384)
}

\begin{abstract}
摘要: 绝缘子是输电线路上的重要设备, 若发生故障会给电力设备带来巨大损失, 从拍摄的输电线路 红外图像中定位和提取绝缘子, 基本上能反映多种绝缘子故障, 在绝缘子的识别和故障诊断中更具实 用性。本文提出了一种基于红外图像的绝缘子提取方法, 依次使用 Speeded Up Robust Features (SURF) 算法提取测试红外图像的关键特征点、基于改进 Fuzzy C-means（FCM）算法聚类划分特征点、根据 绝缘子的形状特征值识别和定位绝缘子、基于改进的图像开运算精确提取绝缘子。该方法充分发挥了 红外图像的优点, 能够准确提取绝缘子, 为基于红外图像的绝缘子故障诊断奠定了基础。
\end{abstract}

关键词: 绝缘子; 红外图像; SURF 特征点; FCM 算法; 图像开运算;

中图分类号：TN219 文献标志码：A 文章编号：1001-8891(2020)09-0840-06

\section{Insulator Extraction Method Based on Infrared Image}

\author{
ZHAO Lihong, GAO Qiang, YU Xiao, LI Dahua \\ (School of Electrical \& Electronic Engineering, and Tianjin Key Laboratory for Control Theory \& Applications in Complicated Systems, \\ Tianjin University of Technology, Tianjin 300384, China)
}

\begin{abstract}
Insulators are important equipment on the transmission line. if fault occurs, it will bring huge loss to power equipments. Locating and extracting insulator from infrared image of transmission line can basically reflect a variety of insulator faults, which is more practical in insulator identification and fault diagnosis.This paper proposes an insulator recognition method based on infrared images, which sequentially use the Speeded Up Robust Features (SURF) algorithm to extract the key feature points of the test infrared images, cluster the feature points based on the improved Fuzzy C-means (FCM) algorithm, identify and locate the insulators according to the shape feature values of the insulators and precise extraction of insulators based on improved image opening operation. This method makes full use of the advantages of infrared images and can accurately extract insulators, which lays a foundation for insulator fault diagnosis based on infrared images.
\end{abstract}

Key words: insulator, infrared image, SURF feature points, FCM algorithm, image opening operation

\section{0 引言}

输电线路检测是保证电网安全可靠运行的重要 工作，绝缘子检测是其中的重要内容之一 ${ }^{[1]}$ 。由于受 地形环境、人员素质、气象条件等因素的影响, 传统 的长周期人工巡线效率低、精度不高 ${ }^{[2]}$ 。近年来, 直 升机和无人机的智能检测正逐步取得进展 ${ }^{[3]}$, 但检测 所获得的数据需要人工处理, 工作人员需要查看大量 的视频和图像来查找故障, 这一过程容易出现问题, 且耗时长 ${ }^{[4]}$ 。

近年来，图像识别技术开始应用于航空影像中的
绝缘子识别, 进而对绝缘子故障进行诊断, 但由于可 见光图像易受天气和输电线路周围环境的影响, 实际 结果不能满足现场的要求 ${ }^{[5]}$, 近年来开始使用红外图 像对绝缘子故障进行检测。

为了提高红外图像目标的提取精度, 国内外学者 提出了多种方法, 主要有阈值 ${ }^{[6]}$ 、马尔可夫随机场 (Markov Random Field) ${ }^{[7]}$ 、主动轮廓模型 ${ }^{[8]}$ 、模糊 c 均值（FCM）聚类 ${ }^{[9]}$ 和神经网络 (Neural Network, $\mathrm{NN})^{[10]}$ 等。文献[11]基于阈值的方法在灰度差较小的 图像中, 由于没有考虑空间信息, 容易产生误分类。 文献[12]基于 MRF 的方法具有严格的数学理论和较 
强的可扩展性, 但是, 它们可能会陷入局部最优, 因 此需要一个合适的初值。文献[13]基于主动轮廓模型 的方法可以自动处理拓扑变化, 具有捕获局部变形的 优点, 但对初始曲线敏感。文献[10]基于神经网络的 方法需要大量的红外图像进行训练, 训练的准确率与 训练涉及的图像数量有关。

本文提出了一种基于红外图像的绝缘子提取方 法, 该方法充分发挥了红外图像的优点, 能够准确提 取绝缘子, 为基于红外图像的绝缘子故障诊断奠定了 基础。首先, 提取图像的 SURF 特征点; 其次, 基于 改进 FCM 算法聚类特征点; 最后, 找到所有相关的 每个类的区域，计算最小外接矩形以及每个区域的形 状特征值以识别绝缘子, 并通过矩形框将其定位; 然 后基于改进的图像开运算精确提取绝缘子。该方法可 作为绝缘子故障检测基于图像处理技术诊断的依据。

\section{1 算法设计}

为了在复杂背景下从航拍图像中提取绝缘子, 提 出一种基于红外图像的绝缘子提取方法。图 1 是该方 法的流程图。

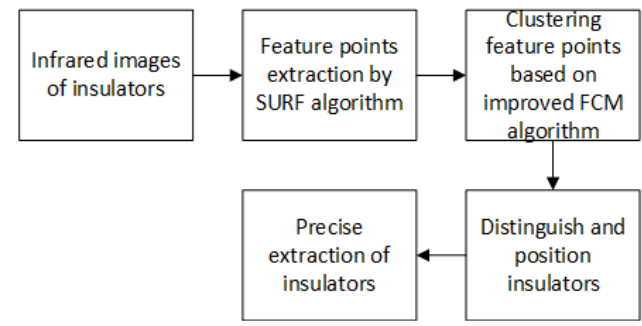

图 1 绝缘子提取流程图

Fig.1 Insulators extraction flow chart

\subsection{SURF 特征点检测}

利用 SURF 算法提取红外图像的 SURF 特征点。 SURF 算法定位 “兴趣点”，这些点是明确的，在局 部和全局扰动下保持稳定, 不随图像的缩放和旋转改 变, 甚至对噪声, 遮挡, 3D 视角转变, 仿射变换和 照明变化都具有鲁棒性。在一定尺度空间水平上计算 积分图像和行列式响应, 对行列式响应图进行尺度归 一化和非极大值抑制, 以便在尺度空间定位 “兴趣 点”。然后将非极大值抑制结果与预先确定的阈值进 行比较。最后, 实现 “兴趣点” 在尺度空间的定位。

\section{2 改进的 FCM 算法}

由于电气设备的存在, 红外图像具有边缘模糊、 电气设备强度突出、噪声污染严重的特点。电气设备 红外图像的特点增加了电气设备故障诊断的难度, FCM 算法是解决这一问题的合适方法。然而, FCM 算法存在非自适应、复杂度高、忽略背景干扰等缺点。 为了弥补这些不足, 提高设备故障检测的准确性, 本
文提出了一种改进的 FCM 算法。

\subsection{1 初始化聚类参数}

原始红外图像对初始聚类中心的敏感性和低对 比度都会影响聚类结果。本文提出一种显著性检测算 法来获取显著性图像, 该算法可以确定聚类中心。将 像素之间的差异度定义为:

$$
d\left(p_{i}, p_{j}\right)=d_{\text {gray }}\left(p_{i}, p_{j}\right) \cdot \exp \left(-\alpha \cdot d_{\text {position }}\left(p_{i}, p_{j}\right)\right)
$$

式中: $d_{\text {gray }}\left(p_{i}, p_{j}\right)$ 是指像素 $p_{i}$ 和 $p_{j}$ 在灰度空间的欧式距 离; $d_{\text {position }}\left(p_{i}, p_{j}\right)$ 是指像素 $p_{i}$ 和 $p_{j}$ 在位置空间的欧式距 离。将 $d_{\mathrm{gray}}\left(p_{i}, p_{j}\right)$ 和 $d_{\mathrm{position}}\left(p_{i}, p_{j}\right)$ 标准化到 $[0,1]$ 。 $\alpha$ 是空 间距离的权重因子。当像素 $p_{i}$ 高度不同于其他所有像 素点, 就认为像素点 $p_{i}$ 是显著点。实际上, 只需要考 虑与中心像素点 $p_{i}$ 最相似的像素。因此, 我们在图像 中以像素 $p_{i}$ 为中心寻找 $K$ 个最相似的像素 $\left\{q_{k}\right\}_{k=1}{ }^{K}$ 。像 素点 $p_{i}$ 的显著值定义为:

$$
S_{i}=\omega\left(p_{i}\right) \cdot \frac{1}{K} \sum_{k=1}^{K} d\left(p_{i}, q_{k}\right)
$$

式中: $\omega\left(p_{i}\right)$ 是空间优先加权项, 定义为:

$$
\omega\left(p_{i}\right)=\exp \left(-9 d_{i}^{2}\right)
$$

式中: $d_{i}$ 是像素 $p_{i}$ 到图像中心的距离。像素坐标标准 化到[0,1]。根据以上步骤, 得到显著性图像。

根据显著性图像将原始图像划分为多个区域，每 个区域可以得到一个直方图。在每个直方图中，根据 最大密度准则确定一个聚类中心，计算方法如下：

$$
K_{i}=\frac{\sum_{j=\text { start }_{i}}^{\text {start }}+I_{i j}}{W}, \quad i=1,2, \cdots, c
$$

式中: $c$ 是聚类的数量。 $W$ 是窗口的大小, 窗口在直 方图上从左向右滑动, 滑动步长为 $p$, start $_{i}$ 是第 $i$ 个 子直方图的起始灰度, $I_{i j}$ 是第 $i$ 个直方图中灰度值为 $j$ 的像素个数。当 $K_{i}$ 为最大值时，第 $i$ 个聚类中心可 以确定为:

$$
\text { Center }_{i}=\operatorname{start}_{i}+\frac{W}{2}, \quad i=1,2, \cdots, c
$$

\subsection{2 具有局部空间约束的 FCM 算法}

模糊聚类算法基于一定准则, 通过最小化目标函 数对未标记数据进行分类。聚类标准函数定义为:

$$
J_{m}(\boldsymbol{U}, V)=\sum_{k=1}^{c} \sum_{i=1}^{n}\left(u_{k i}\right)^{m}\left(d_{k i}\right)^{2}
$$

式中: $c$ 是聚类的数量; $m$ 是加权指数, 可以控制聚 类结果的模糊度; $n(n=M \times N)$ 是图像的总像素; $u_{k i}$ $\in[0,1]$ 是属于第 $k$ 个聚类的第 $i$ 个像素的模糊隶属度。 所有像素的隶属度可以构成一个矩阵 $\boldsymbol{U}=\left[u_{k i}\right]$, 矩阵 的大小是 $c \times n$ 。 $d_{k i}=\left\|x_{i}-v_{k}\right\|$ 是指第 $i$ 个像素到第 $k$ 个 聚类中心的欧氏距离。 $V$ 是聚类中心的集合。 
在目标函数中引入提高局部邻域像素相似度度 量水平的智能控制因子 $G_{k i}$, 并将其定义为:

$$
\begin{gathered}
G_{k i}=\sum_{\substack{j \in N_{i} \\
i \neq j}} \frac{1}{d_{i j}+1}\left(1-u_{k j}\right)^{m} D_{k i} \\
D_{k i}=\left\|x_{i}-v_{k}\right\|^{2}
\end{gathered}
$$

式中: $x_{i}$ 是位于窗口 $\left(N_{i}\right)$ 的邻域像素; $D_{k i}$ 是第 $i$ 个像 素和第 $k$ 个聚类中心之间的距离。 $v_{k}$ 是第 $k$ 个聚类的 聚类中心。 $d_{i j}$ 表示第 $i$ 个像素和第 $j$ 个像素之间的欧 氏距离。

本文中, 采用灰度表示像素来压缩数据空间。在 预处理阶段, 计算每个像素所属的灰度。在每次迭代 中只需要计算一次灰度, 从而降低了模糊聚类方法的 计算复杂度。

为了以较少的迭代次数实现算法的收敛, $D_{k i}$ 重新 定义为:

$$
D_{k i}{ }^{\prime}=\left\|x_{i}^{2}-v_{k}^{2}\right\|
$$

提出一种新的局部空间约束, 定义为:

$$
G_{k i}^{\prime}=\sum_{\substack{j \in N_{i} \\ i \neq j}} \frac{1}{d_{i j}+1}\left(1-u_{k j}\right)^{m} D_{k i}^{\prime}
$$

像素的模糊隶属度发生显著变化, 加快了算法的 收敛性。将灰度和局部空间约束 $G_{k i}{ }^{\prime}$ 引入到目标函数 中, 将改进的 FCM 定义为:

$$
J_{m}(U, V)=\sum_{k=1}^{c} \sum_{i=1}^{N}\left[u_{k i}^{m} D_{k i}^{\prime} h(i)+\sum_{\substack{j \in N_{i} \\ i \neq j}} \frac{1}{d_{i j}+1}\left(1-u_{k j}\right)^{m} D_{k i}^{\prime}\right]
$$

式中: $N$ 是图像中灰度的总数; $h(i)$ 是红外图像的直方 图; $i=0,1, \cdots, L-1$ 表示图像灰度级; $N_{i}$ 代表以灰度 为 $i$ 的像素为中心的 8 邻域窗口; $j$ 是 $N_{i}$ 中的一个像 素点。这种改进可以有效降低计算复杂程度。

\section{3 识别和定位绝缘子}

绝缘子的形状特征值有细长度、圆形度、占空比 和 $H_{\mathrm{u}}$ 不变矩。从拍摄的绝缘子红外图像中, 随机选 取 40 张, 本文计算细长度和占空比两个特征值。

\section{1) 细长度 $B_{\text {lwratio }}$}

细长度是指该区域的最小外接矩形的纵横比。细 长度的计算公式如下:

$$
B_{\text {lwratio }}=\frac{B_{\text {length }}}{B_{\text {widh }}}
$$

式中: $B_{\text {length }}$ 和 $B_{\text {width }}$ 分别为最小外接矩形的长和宽。

图 2 为选取的绝缘子红外图像中, 绝缘子和背景 区域的最小外接矩形的细长比。由图可知, 绝缘子的
细长比在[2.1386, 7.9532]之间, 而背景区域细长度则 普遍较小。

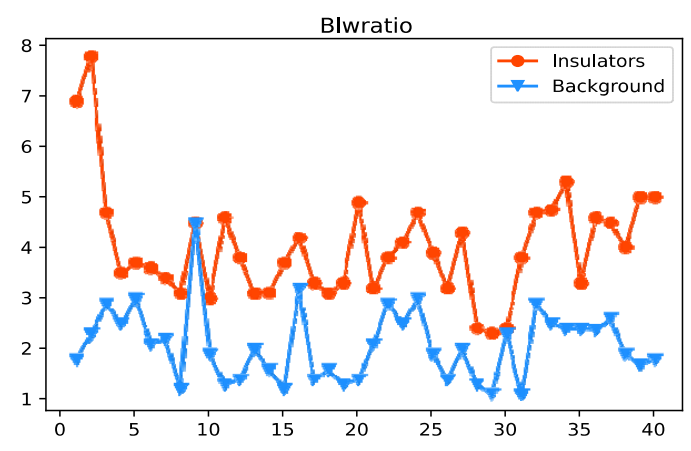

图 2 各区域的细长度

Fig.2 Slender length of each area

2）占空比 $Z_{\text {dutyfactor }}$

占空比是指目标区域占外接矩形的比例。占空比 的计算公式如下:

$$
Z_{\text {dutyfactor }}=\frac{F_{\text {area }}}{S_{\text {boundingbox }}}
$$

式中: $F_{\text {area }}$ 表示目标区域的面积; $S_{\text {boundingbox }}$ 表示区域 外接矩形的面积。

图 3 显示了选取的绝缘子红外图像中, 绝缘子和 背景区域的占空比。图中绝缘子的占空比在 [0.5261, 0.7936]之间, 背景区域具有宽范围的占空比变化, 很 强的随机性, 并且大多数都低于绝缘子。

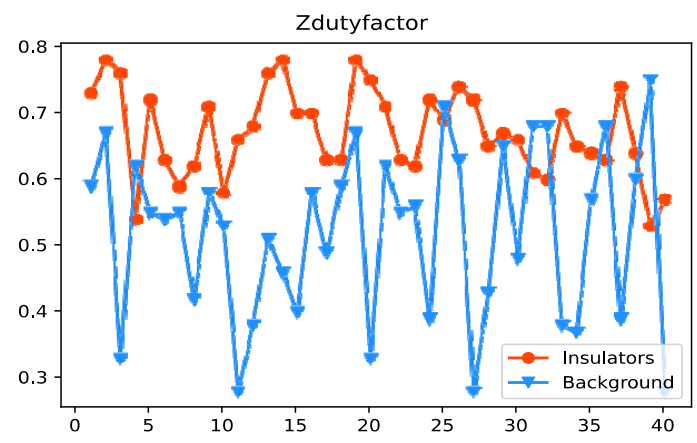

图 3 各区域的占空比

Fig.3 Duty cycle of each area

由以上分析可知, 一般情况下绝缘子的细长比和 占空比大于塔架, 本文利用这两个指标来找出绝缘 子。利用改进的 FCM 算法聚类特征点, 聚类的连通 区域视为可疑目标。如果一个聚类的形状特征值满足 条件, 则可以将其定义为绝缘子。因此, 所有区域都 可以获得并计算最小外接矩形和每个区域的形状特 征值来识别绝缘子。用长方形标记绝缘子, 所有的绝 缘子可以被识别和定位出来。

\section{4 绝缘子的精确提取}


绝缘子故障检测需要从图像中提取出纯粹的绝 缘子串图像。在确定绝缘子区域后, 从中提取最大连 通域。使用绝缘子区域定位后, 即使图像主要部分仍 然是塔架和电线, 也可以精确地分离绝缘子区域。尽 管定位效果好, 但通过传统方法获得的图像中仍有不 属于绝缘子部分如电线和一些小的结构, 这会干扰分 割结果, 从而降低了结果的质量和效果。

图像的开运算可以达到断开细小连接并去除图 像边缘上的毛刺的效果。图像开运算是图像形态处理 的基本操作, 是先腐蚀目标图像然后进行膨胀的过 程。

本文提出一种改进的图像开运算方法。

1) 选用较小的结构元素来腐蚀图像, 以减少图 像本身的变化, 还可以消除干扰。

2）提取最大连通域。

3) 膨胀连通域, 避免由于结构太小导致的本应
分离的结构发生重新连接。

即在图像的开运算过程中, 在腐蚀和膨胀之间, 加入提取最大连通域。

\section{2 实验结果与分析}

\section{1 实验结果}

为了验证该方法的有效性, 做了大量实验。但考 虑到空间的限制, 仅详细列举了其中一张绝缘子红外 图像的提取过程如图 4。

本文选取 4 组不同场景下拍摄的绝缘子红外图 像，从中提取绝缘子区域。在聚类特征点时，分别采 用传统 FCM 算法、K-means 算法、均值漂移聚类算 法、DBSCAN (Density-Based Spatial Clustering of Applications with Noise）算法提取绝缘子最终结果和 本文算法进行对比实验, 实验结果如图 5 所示。

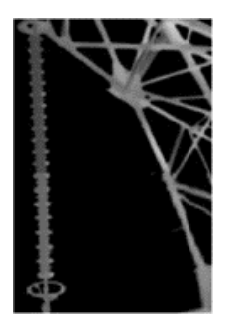

(a) 红外图像灰度图

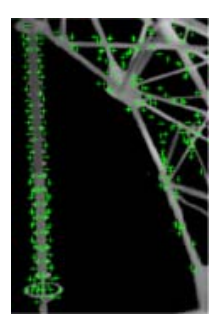

(b) SURF 特征点

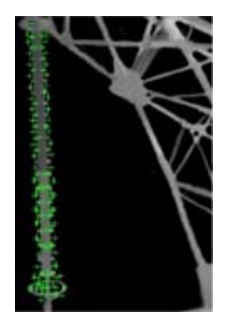

(c) 绝缘子聚类

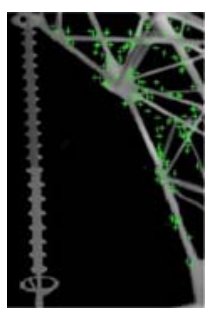

(d) 杆塔聚类

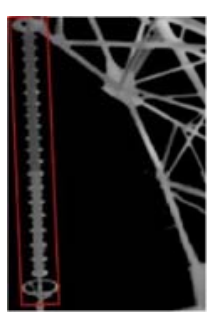

(e) 绝缘子定位

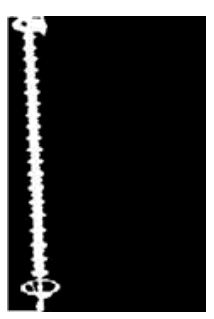

(f) 最大连通域

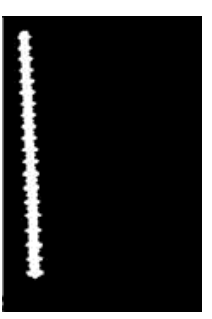

(g) 精确绝缘子

(a) Infrared image grayscale (b)SURF feature points

(c)Insulator clustering

(d)Tower clustering

(e) Insulator positioning (f) Maximum connected domain (g) Precision insulator

图 4 绝缘子图片

Fig.4 Insulator pictures

(a) Original images

(b) Our algorithm
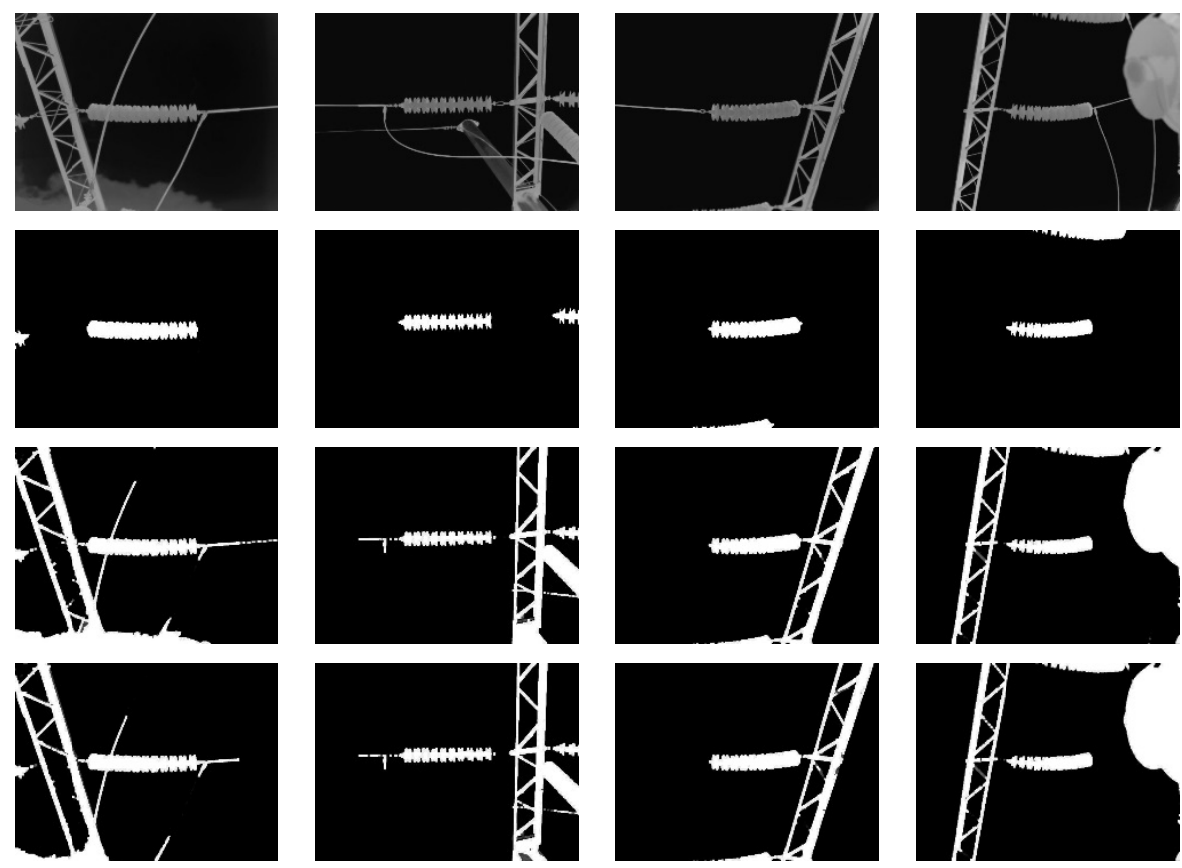
(e) Mean shift clustering algorithm
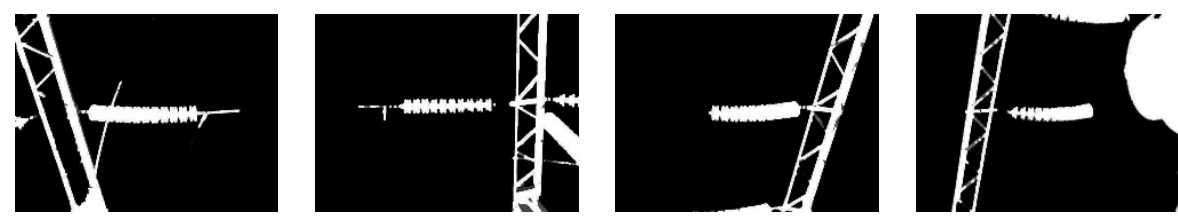

(f) K-means algorithm
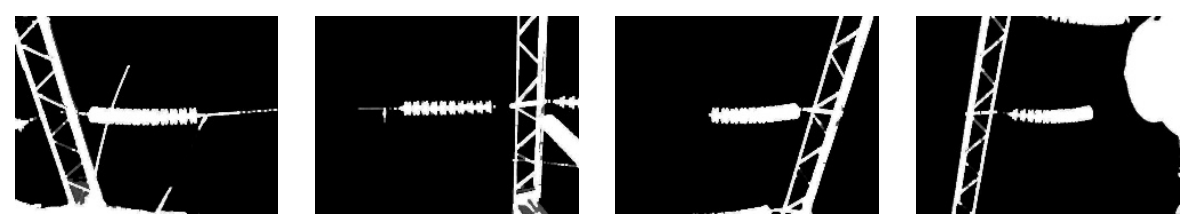

图 5 实验结果对比图

Fig.5 Experimental results comparison chart

由实验结果对比可以定性地分析出, 在绝缘子红 外图像中, 本文算法可以准确获得绝缘子的完整形 态, 提取出纯粹绝缘子串。传统 FCM 算法、K-means 算法、均值漂移聚类算法、DBSCAN 算法在聚类特征 点时, 效果不好, 导致绝缘子定位失败, 最终提取效 果不好。虽也提取到完整的绝缘子区域, 但也提取出 大量背景区域, 提取纯粹绝缘子失败。

\section{2 实验分析}

本文选择真阳率（TPR），假阳率（FPR）和准 确率 (ACC) 作为各类算法结果对比的指标, 公式如 下:

$$
\begin{gathered}
\mathrm{TPR}=A_{\mathrm{t}} / T \\
\mathrm{FPR}=B_{\mathrm{f}} / P \\
\mathrm{ACC}=\frac{A_{\mathrm{t}}+B_{\mathrm{t}}}{T+P}
\end{gathered}
$$

式中: $A_{\mathrm{t}}$ 表示实验提取绝缘子结果与标准参考图像目 标点一致的像素点数; $T$ 表示参考绝缘子的目标区域; $B_{\mathrm{f}}$ 表示实验提取结果中应是背景部分但被误分为绝 缘子的像素点数; $B_{\mathrm{t}}$ 表示实验提取结果与参考图像背 景点一致的像素点数; $P$ 表示参考图像的背景区域。

实验中所用各类算法对 4 幅原图的检测结果指标 数值如表 1 所示。

由表 1 可以看出, 本文算法在达到真阳率较高的 同时, 也保证了假阳率较低, 可以准确地完成绝缘子 目标区域的提取，而其他算法达到了真阳率高的同 时, 没有保证较低的假阳率, 表示提取到的绝缘子区 域面积过大, 除了绝缘子串, 还包含较多背景区域。

由以上分析可知, 本文算法所提取到的绝缘子更 为准确, 几乎没有背景区域的干扰, 与真实绝缘子区 域的重合度较高。

\begin{tabular}{|c|c|c|c|c|c|c|}
\hline \multicolumn{3}{|l|}{ order } & FCM & K-means & Mean shift & DBSCAN \\
\hline \multirow{3}{*}{1} & TPR & 0.9777 & 0.9797 & 0.9725 & 0.9621 & 0.9761 \\
\hline & FPR & 0.0011 & 0.1236 & 0.0903 & 0.0764 & 0.1048 \\
\hline & ACC & 0.9982 & 0.8798 & 0.9117 & 0.9248 & 0.8978 \\
\hline \multirow{3}{*}{2} & TPR & 0.9608 & 0.9563 & 0.9259 & 0.8898 & 0.9455 \\
\hline & FPR & 0.0014 & 0.0923 & 0.0844 & 0.0790 & 0.0878 \\
\hline & ACC & 0.9978 & 0.9087 & 0.9158 & 0.9203 & 0.9128 \\
\hline \multirow{3}{*}{3} & TPR & 0.9883 & 0.9704 & 0.9812 & 0.9772 & 0.9856 \\
\hline & FPR & 0.0009 & 0.0766 & 0.0707 & 0.0693 & 0.0736 \\
\hline & ACC & 0.9987 & 0.9250 & 0.9307 & 0.9319 & 0.9279 \\
\hline \multirow{3}{*}{4} & TPR & 0.9641 & 0.9312 & 0.9732 & 0.9075 & 0.9274 \\
\hline & FPR & 0.0005 & 0.1639 & 0.1841 & 0.1606 & 0.1170 \\
\hline & ACC & 0.9987 & 0.8381 & 0.8191 & 0.8408 & 0.8839 \\
\hline \multicolumn{2}{|c|}{ TPR means } & 0.9727 & 0.9594 & 0.9632 & 0.9342 & 0.9587 \\
\hline \multicolumn{2}{|c|}{ FPR means } & 0.0010 & 0.1141 & 0.1074 & 0.0963 & 0.0958 \\
\hline \multicolumn{2}{|c|}{ ACC means } & 0.9984 & 0.8879 & 0.8943 & 0.9044 & 0.9056 \\
\hline
\end{tabular}

表 1 不同算法定量分析对比

Table 1 Quantitative analysis and comparison of different algorithms 


\section{3 结论}

本文介绍了一种基于红外图像的绝缘子提取方 法。首先, 使用 SURF 提取测试红外图像的关键特征 点, 然后根据基于改进 FCM 聚类算法将特征点划分 为 $k$ 个互斥类。找到每个类别的连接区域, 并计算最 小外接矩形和每个区域的形状特征值以识别绝缘子, 并通过矩形框定位绝缘子。最后, 利用在图像的开运 算过程中穿插最大连通域提取的融合方法, 精确提取 绝缘子。在测试的绝缘子红外图像中, 本文通过实验 结果定量分析验证了这种方法的有效性。

\section{参考文献:}

[1] 王沝, 杜伟, 孙鸿博, 等. 基于红外图像识别的输电线路故障诊断方 法[J]. 红外技术, 2017, 39(4): 383-386.

WANG Miao, DU Wei, SUN Hongbo, et al. Transmission Line Fault Diagnosis Method Based on Infrared Image Recognition[J]. Infrared Technology, 2017, 39(4): 383-386.

[2] 刘正庭, 尹骏刚, 李凯迪, 等. 基于分水岭算法的绝缘子串红外图像 分割方法[J]. 电瓷避雷器, 2020(2): 216-221, 228.

LIU Zhengting, YIN Jungang, LI Kaidi, et al. Infrared Image Segmentation of Insulator Strings Based on Watershed Algorithm[J]. Insulators and Surge Arresters, 2020(2): 216-221, 228.

[3] 付强, 姚建刚, 李唐兵, 等. 基于红外图像的绝缘子串钢帽和盘面区 域自动提取方法[J]. 红外技术, 2016, 38(11): 969-974, 979.

FU Qiang, YAO Jiangang, LI Tangbing, et al. The Automatic Extraction Method of the Insulator String's Steel Capsand Disks Area Based on Infrared Image[J]. Infrared Technology, 2016, 38(11): 969-974, 979.

[4] 张晓春, 欧阳广泽, 何洪英, 等. 基于红外图像匹配的零值绝缘子检 测 [J]. 电测与仪表, 2019, 56(6): 100-105.

ZHANG Xiaochun, OUYANG Guangze, HE Hongying, et al. Zero-insulator detection based on infrared images matching[J].
Electrical Measurement \& Instrumentation, 2019, 56(6): 100-105.

[5] 张也，彭子健，付强，等. 环境湿度对瓷质绝缘子串电压分布及红外 热像检测的影响分析[J]. 电网技术, 2018, 42(4): 1342-1349.

ZHANG Ye, PENG Zijiang, FU Qiang, et al. Analysis of Environment Humidity Influence on Voltage Distribution and Infrared ThermalImage Detection of Porcelain Insulator Strings[J]. Power System Technology, 2018, 42(4): 1342-1349.

[6] Oliveira J P S de, Conci A, Pérez M G, et al. Segmentation of infrared images: A new technology for early detection of breast diseases[C]//IEEE International Conference on Industrial Technology, Seville, 2015: 1765-1771.

[7] ZHAO Q, Xiao-li L, YU L, et al. A fuzzy clustering image segmentation algorithm based on Hidden Markov Random Field models and Voronoi Tessellation[J]. Pattern Recognition Letters, 2017, 85(1): 49-55.

[8] ZHANG R, ZHU S, ZHOU Q. A novel gradient vector flow snake model based on convex function for infrared image segmentation[J]. Sensors, 2016, 16(10): 1756.

[9] BAI X,WANG Y, GUO S. Symmetry Information Based Fuzzy Clustering for Infrared Pedestrian Segmentation[J]. IEEE Transactions on Fuzzy Systems, 2018, 26(4): 1946-1959.

[10] ZHANG H, HONG X. Infrared image segmentation for photovoltaic panels based on Res-UNet[C]//IEEE Pattern Recognition and Computer Vision, 2019: 611-622.

[11] HE L, HUANG S. Modified firefly algorithm based multi-level thresholding for color image segmentation[J]. Neurocomputing, 2017, 240(5): 152-174.

[12] YANG X, GAO X, TAO D, et al. An efficient MRF embedded level set method for image segmentation[C]//IEEE Transactions on Image Processing, 2015, 24(1): 9-21.

[13] NIU S, CHEN Q, JI Z, et al. Robust noise region-based active contour model via local similarity factor for image segmentation[J]. Pattern Recognition, 2017, 61(7): 104-119. 